\title{
A point mutation in Phytochromobilin Synthase alters the circadian clock and photoperiodic flowering of Medicago truncatula
}

\author{
Soledad Perez Santangelo ${ }^{1}$, Nathanael Napier ${ }^{2}$, Fran Robson ${ }^{3}$, James Weller ${ }^{4}$, Donna \\ Bond $^{1}$, and Richard Macknight ${ }^{5}$ \\ ${ }^{1}$ University of Otago Division of Health Sciences \\ ${ }^{2}$ Plant and Food Research Palmerston North \\ ${ }^{3}$ University of Bristol School of Biological Sciences \\ ${ }^{4}$ University of Tasmania \\ ${ }^{5}$ University of Otago
}

September 25, 2021

\begin{abstract}
Plants use seasonal cues to initiate flowering at an appropriate time of year to ensure optimal reproductive success. The circadian clock integrates these daily and seasonal cues with internal cues to initiate flowering. The molecular pathways that control the sensitivity of flowering to photoperiod (daylength) are well described in the model plant Arabidopsis. However, much less is known in crop species, such as the legume family species. Here we performed a flowering time screen of a TILLING population of Medicago truncatula and found a line with late-flowering and altered light-sensing phenotypes. Using RNA-sequencing, we

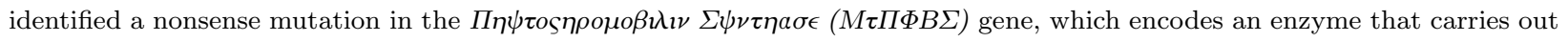
the final step in the biosynthesis of the chromophore required for phytochrome $(P H Y)$ activity. The analysis of the circadian clock in the Mtp $\Phi$ bs mutant revealed a shorter circadian period, which was shared with the phyA mutant. The $M \tau \pi \Phi \beta \varsigma$ and MtphyA mutants showed downregulation of FT floral regulators MtFTa1, MtFTb1/b2 and a shift in phase for morning and night core clock genes. Our findings show that PHYA is necessary to synchronize the circadian clock and integration of light signaling to promote expression of the $M t F T$ genes to precisely time flowering.
\end{abstract}

\section{Hosted file}

MS_PerezSantangeloMtpobs_6Sep21.docx available at https : //authorea.com/users/433090/articles/ 539016-a-point-mutation-in-phytochromobilin-synthase-alters-the-circadian-clock-andphotoperiodic-flowering-of-medicago-truncatula

\section{Hosted file}

Figures_Sep2021.pdf available at https://authorea.com/users/433090/articles/539016-a-pointmutation-in-phytochromobilin-synthase-alters-the-circadian-clock-and-photoperiodicflowering-of-medicago-truncatula 